\title{
Immediate microvascular maxillofacial reconstruction and dental rehabilitation: protocol, case report, and literature review
}

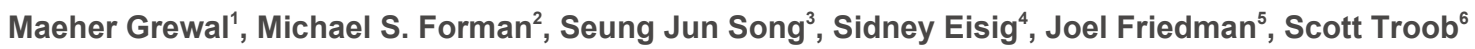 \\ 'Department of Otolaryngology-Head and Neck Surgery, Columbia University Vagelos College of Physicians and Surgeons, New \\ York, NY 10032, USA. \\ ${ }^{2}$ Department of Oral and Maxillofacial Surgery, NewYork Presbyterian Hospital, Columbia University Medical Center, New York, \\ NY 10032, USA. \\ ${ }^{3}$ Division of Prosthodontics, Columbia University College of Dental Medicine, New York, NY 10032, USA. \\ ${ }^{4}$ Department of Oral and Maxillofacial Surgery, Section of Hospital Dentistry, New York Presbyterian Hospital/Columbia \\ University Irving Medical Center, Columbia University College of Dental Medicine, New York, NY 10032, USA. \\ ${ }^{5}$ Department of Oral and Maxillofacial Surgery, Columbia University College of Dental Medicine, New York, NY 10032, USA. \\ ${ }^{6}$ Department of Otolaryngology-Head and Neck Surgery Columbia University Irving Medical Center, New York, NY 10032, USA.
}

Correspondence to: Dr. Scott Troob, Department of Otolaryngology-Head and Neck Surgery Columbia University Irving Medical Center, 180 Fort Washington Ave, New York, NY 10032, USA. E-mail: sht22@cumc.columbia.edu

How to cite this article: Grewal M, Forman MS, Song SJ, Eisig S, Friedman J, Troob S. Immediate microvascular maxillofacial reconstruction and dental rehabilitation: protocol, case report, and literature review. Plast Aesthet Res 2021;8:37.

https://dx.doi.org/10.20517/2347-9264.2021.43

Received: 4 May 2021 First Decision: 26 May 2021 Revised: 4 Jun 2021 Accepted: 16 Jun 2021 First online: 6 Jul 2021

Academic Editors: Matthew Spector, Mark K. Wax Copy Editor: Xi-Jun Chen Production Editor: Xi-Jun Chen

\begin{abstract}
Maxillomandibular ablation and reconstruction includes a series of procedures that can be disfiguring and emotionally traumatic for a patient while treating maxillofacial disease. Often times, the process of regaining full form and function may take months to years for a patient. With the advent of modern day technology and virtual surgical planning, some patients may benefit from a single operation that includes disease ablation, microvascular reconstruction, and immediate dental rehabilitation, also known as "Jaw in a Day".
\end{abstract}

Keywords: Endosseous implants, free fibula flap, immediate reconstruction, Jaw in a Day, mandibular reconstruction, maxillary reconstruction, virtual surgical planning 


\section{INTRODUCTION}

Historically, maxillomandibular ablation and reconstruction include a series of intensive procedures aimed at removing lesions of either the maxilla or mandible and then reconstructing the jaws to appropriate form and function. This dental rehabilitation post-fibula free flap reconstruction was staged and took months to years to yield the final product, if completed at all. The defects in the meantime are often disfiguring, affecting their facial appearance and their ability and desire to perform daily functions such as eat, smile, or communicate. Such a complex operation requires not only assembly of and coordination between multiple surgical teams, but also meticulous selection of the patient candidate and precise planning of each step of the surgical workflow. Restoration of function is often dependent on reconstruction of the occlusion. Precision is of utmost importance since patients with native teeth can detect differences as low as 8 to 10 microns.

Often several additional procedures were required to debulk scar tissue and allow for sufficient healing of the fibula-mandible or fibula-maxilla bony union. Only once the flap was healed were dental implants (DIs) placed into the fibula for eventual delivery of a dental prosthesis. DIs heal by osseointegration which typically requires 3-4 months before they can be loaded with a dental prosthesis. Therefore, appropriate form and function for a patient, by means of dental prostheses, were not delivered until the graft had been fully integrated and the DIs integrated into the fibula graft, typically 6-9 months in total. Radiation therapy can delay this even further.

However, maxillofacial reconstruction via microvascular free flaps have seen tremendous progress in the past decade since the advent of the "Jaw in a Day" (JIAD) procedure. "Jaw in a Day" represents the process of a single operation which includes: ablation of maxillary or mandibular lesion, immediate reconstruction with a microvascular free flap, immediate DI placement into the graft, and immediate delivery of dental prosthesis. In 2013, an interdisciplinary team of surgeons described harnessing the breakthrough technology of virtual surgical planning to achieve dental rehabilitation to the reconstructed mandible with a fibula free flap simultaneously in four patients ${ }^{[1]}$.

In 2016, an additional institution reported on three patients in whom they used this same technique ${ }^{[2]}$. Both reported on patients with benign masses of the mandible and maxilla that required no further radiation therapy post-surgery that might have affected osseointegration. Since then, several other institutions have described their successes in fibular-mandible and fibular-maxilla reconstruction, although in albeit a smaller number of patients ${ }^{[1-8]}$. These studies emphasize the both the feasibility and room for expanded application of such techniques, which carries the potential to leverage technology to reduce the burden of surgery on a vulnerable patient population.

Virtual surgical planning (VSP) improves three-dimensional accuracy of the reconstruction and provides detailed calculations and spatial configurations needed to ensure successful fit of the graft, and occlusion of the reconstructed jaw. It allows for specific measurements of the patient's maxilla or mandible and fibulas to a print custom reconstruction plate and surgical cutting guide unique to each patient's specific osteotomies. Utilizing VSP reduces both human error and surgical time, making JIAD possible at the utmost benefit to patients.

\section{PROTOCOL}

Successful "Jaw in a Day" surgery requires extensive planning and preparation with an experienced multidisciplinary team. Briefly, once an appropriate patient has been identified and the team assembled, a virtual surgical planning session is held to plan all aspects of the resection and reconstruction, and establish 
an intraoperative workflow with an emphasis on efficiency and patient safety [Figure 1].

\section{General considerations: patient population, team assembly, and financial plan}

Patient population

Patients with benign jaw disease localized to the maxilla or mandible who are motivated to have immediate dental tooth replacement have the most to be gained from this procedure. The ideal JIAD candidate patient is one with benign disease such as an odontogenic keratocyst (OKC), ameloblastoma, odontogenic myxoma, or medication-related osteonecrosis of the jaw (MRONJ), a stable existing occlusion, and high post-operative expectations. Additionally, good oral hygiene and intact dentition is a must; this procedure is less successful in recreating dentition or occlusion already lost to disease. Patients with oncologic disease processes that require post-operative radiation are less favorable as radiation can damage bone mineralization, reducing osteointegration of the implants. However, several centers have expanded the application to patients with malignancies, without increased rate of complications ${ }^{[4]}$.

Once a patient has been selected, a multidisciplinary team with clearly defined roles is established. The team consists of an ablative surgeon, a microvascular trained reconstructive surgeon [either Otolaryngology Head and Neck Surgery, Oral and Maxillofacial Surgeon (OMS), or Plastic Surgeon], and an appropriately trained prosthodontist, usually a maxillofacial prosthodontist. The surgeons are responsible for ablation of the disease, harvesting and inset of a vascularized free flap, and closure of the head and neck incisions. An OMS assists with the ablation and inset, and completes the implant placement. Finally, a prosthodontist designs the prosthesis, loads the prosthesis to the DIs, and oversees postoperative modifications to ensure proper occlusion and function of the maxillary and mandibular teeth. Regarding the post-operative oral rehabilitation and functional occlusion, a dedicated prosthodontist is critical to procedural success. Practitioners must be aware of the length, complexity and multidisciplinary nature of the operation; they should be willing to undertake the significant risks of a time intensive surgical procedure and should be experts in their respective fields.

This procedure can be a significant financial burden to the patient. Often times, as medical insurance does not cover dental rehabilitation, the patient will incur most of the dental related expenses which may include the placement of DIs and almost always includes the cost of the provisional and definitive prostheses. The cost of the implants themselves can be bundled with hospital materials management. However, the costs of the provisional acrylic prosthesis, all related components, and the definitive prosthesis of zirconia or porcelain can still approach tens of thousands of dollars. While fees may vary per geographic region, these fees are comparable to traditional prosthetic work that requires precious materials and high laboratory fees. The patient should be counselled as to a feasible financial plan for affording the operation and dental treatment options.

\section{Preoperative preparation}

Ablation and reconstruction

Before proceeding with the operation, the team will need to evaluate the location and extent of the patient's pathology as well as the condition of their existing dentition. The patient will also need a lower extremity CT angiogram to evaluate patency of the peroneal vessels and adequate three-vessel run-off to determine the best candidate leg for graft harvest.

\section{Prosthodontist consult}

Data collection

The prosthodontist will obtain intraoral and extraoral preliminary photos for documentation and evaluation purposes. Either an intraoral scan or a conventional impression is taken to produce a digital or 


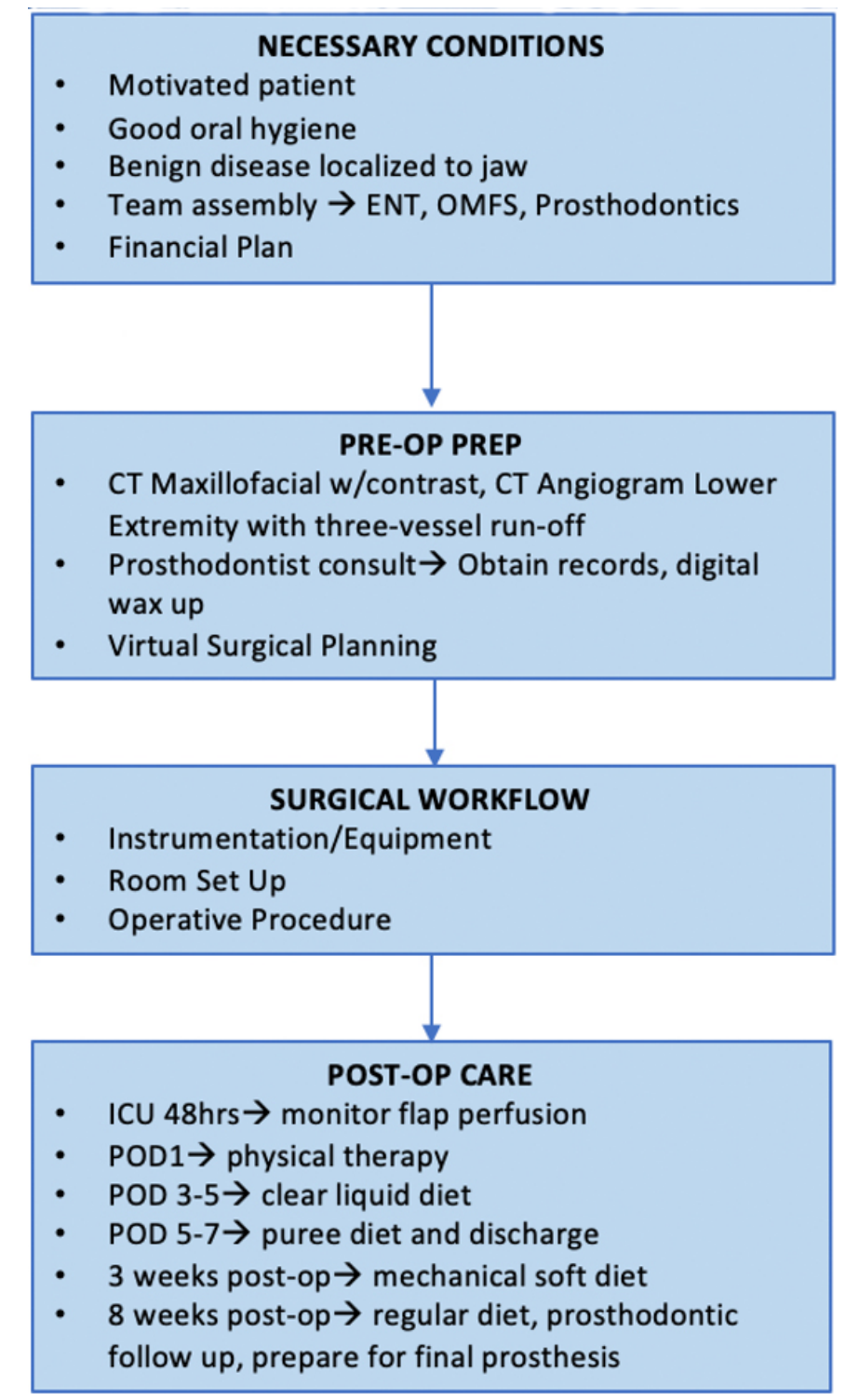

Figure 1. Protocol workflow for maxillomandibular ablation, immediate reconstruction with microvascular free flap, and immediate dental implant and prosthesis placement.

stone cast respectively. In the case of a conventional impression and stone cast, an additional step to digitize the cast via laboratory scan is necessary. If the patient has an edentulous area, a digital or conventional waxup (emulation of tooth form and position with wax) will be done and uploaded to VSP for treatment planning purposes [Figure 2].

\section{Provisional prosthesis fabrication}

Following VSP, a provisional restoration will be fabricated from polymethyl methacrylate (PMMA) with the proposed DI position in consideration. The provisional restoration will be milled from a PMMA block for adequate structural strength with a convex hygienic design. During the operation the provisional prosthesis will be engaged to the DIs for optimal occlusal alignment and then disengaged for polishing. Following completion, the provisional prosthesis will be engaged to the DIs for delivery. 


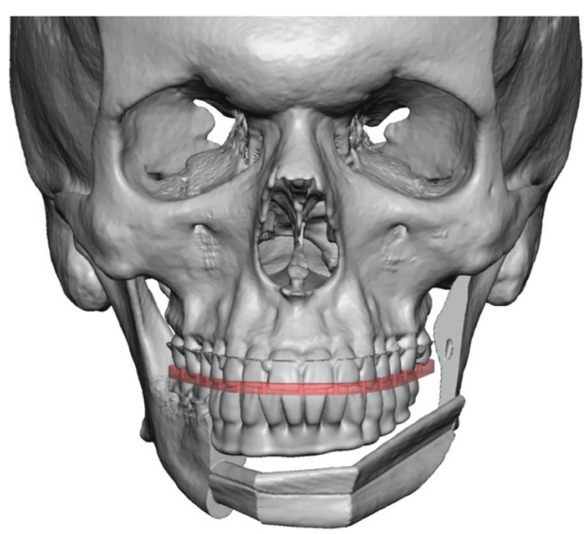

Figure 2. Virtual surgical planning representation of digital provisional and occlusion. In this case, a full wax-up was not needed since the patient had existing dentition, which was suitable and used as a reference.

\section{Definitive prosthesis fabrication}

A definitive prosthesis will be fabricated and delivered once osseointegration of the DIs and soft tissue healing/maturation is confirmed. A definitive prosthesis can be made from zirconia or titanium superstructure with porcelain. Hygienic design is of utmost importance.

\section{Virtual surgical planning}

In the VSP, the surgeons will collaborate and design the osteotomies to excise the disease. This plan will also include the specific design of the cuts on the fibula. Further, the team will plan the location of the DIs to which the dental prosthesis will be attached. The selected locations need to be appropriately spaced throughout each fibular segment. More specifically, the locations of the DIs cannot interfere with planned sites for fixation screws from the reconstruction plate [Figure 3]. It is imperative to ensure the height and width of the fibula can accommodate the implant without fenestration for optimal placement. Ideally, there should be at least 1-2 mm of surrounding bone on either side of the fixture. The sizes of DIs vary between manufactures, but in general range from 3-6 $\mathrm{mm}$ in width and 6-16 $\mathrm{mm}$ in length. DIs are cylindrical in nature and can be parallel or tapered requiring the shape of the fibula triangle (in cross section), to be rotated such that a broad flat side is cephalad to accommodate the width of the implants.

The surgeons and prosthodontist work backwards from where the proper occlusion of the reconstructed jaw needs to be. For example, if the plan is to resect a portion of the right mandible, then the teeth for that segment will be designed (and limited) to appropriately function against the opposing maxillary dentition. Based on the entire surgical simulation using an extensive VSP, maxillary or mandibular and fibular cutting guides, a titanium reconstruction plate, as well as a surgical guide for the DIs are manufactured uniquely for the patient's surgery.

\section{Surgical workflow}

\section{Instrumentation and equipment}

The VSP session, as described above, provide the maxillomandibular and fibula cutting guides and direct the creation of the provisional dental prosthesis. A dental implant system chosen by the OMS and prosthodontist will be required and used to prepare the osteotomies for the DIs that will support the provisional and definitive prostheses. The DI drills follow a protocol of three to five successively larger drills starting from $2.0 \mathrm{~mm}$ up to $5.0 \mathrm{~mm}$ at a speed of 600-1200 rpm (per manufacture guidelines). Copious irrigation throughout this sequence is critical to prevent overheating of the fibula, local necrosis and failure of DI osseointegration. The fibula cutting guides can be designed to include a surgical guide for DI 
A
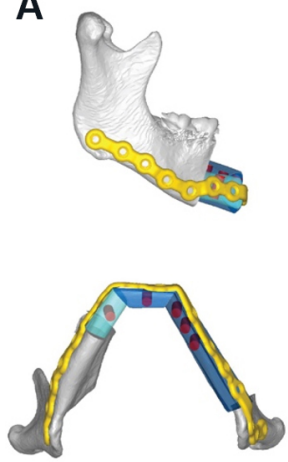
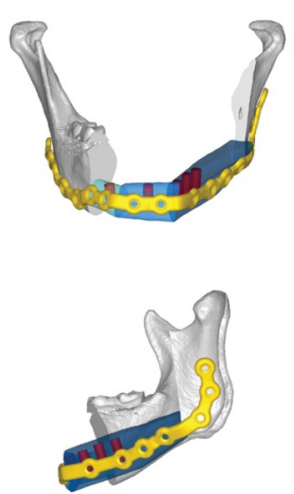

B

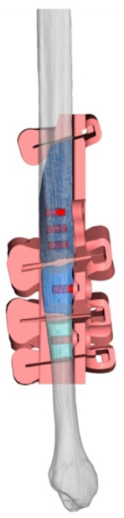

Figure 3. $(A+B)$ Custom plate design and accommodation of dental implants taken into account with the fibula cutting guide, predictive holes, and implant surgical guide. This was all done based on the pre-determined occlusion. A: The 4 Jaw images with prosthesis in yellow in a square layout; B: the fibular image with the guide in pink on the right side.

placement which accommodates guiding keys and drills to minimize angular and positional deviation during preparation of the osteotomies for DI placement. In order to load (engage) the DIs on the day of surgery, primary stability of the DIs is of paramount importance. Typically, each DI should have primary stability that exceeds 35 Newton centimeters $(\mathrm{N}-\mathrm{cm})$ of torque. It is also important to understand that the torque at time of placement should not exceed $70 \mathrm{~N}-\mathrm{cm}$ which increases the risk of localized fracture of the fibula and/or fracture of the DI.

A dental prosthetic component called an intermediary abutment (IA) is also required. These components are made at 0,17 , and 30 degrees and are able to correct for skew, to align all the fixtures and create a path of draw for the prosthodontist to deliver the dental prosthesis, as these components are available in various heights, an additional advantage is elevation of the restorative platform for increased accessibility. A printed model (complete/partial) of the patient's skull is used to ensure proper seating and occlusion of all these various parts during the operation, and a custom titanium reconstruction plate will be used to secure the free flap into the maxilla or mandible.

\section{Room set up}

Once the patient has been induced and intubated with a nasal RAE endotracheal tube by the anesthesia team, the patient is rotated 180 degrees. This allows for enough space to accommodate both sets of surgical teams and simultaneous operation. At our institution, the OMS team begins in the head and neck area while the ENT team begins with the fibular dissection. Two scrub teams are required with two sets of instruments, one dedicated for the head and neck, and one dedicated for the fibula. Additionally, a DI and prosthetic setup will be required since much of this work will be done in situ at the fibula. Since the implants are placed in situ, a skull model with the opposing dental arch is critical for the prosthodontic team to use to refine the provisional prosthesis. This limits manipulation of the flap after inset and microvascular anastomosis at the head and neck.

\section{Operative procedure}

The ablative surgeon makes a mastoid to mastoid visor-shaped incision $2 \mathrm{~cm}$ below the inferior border of the mandible and dissects through the skin and platysma. A combination of sharp and blunt dissection should be used with diligent nerve monitoring to preserve the branches of the seventh cranial nerve. When the facial arteries are encountered, they are clipped, divided and preserved for later anastomosis. As the pterygo-masseteric sling is approached, it is incised carefully to expose the mandible underneath and divide 
away the periosteum. After further exposure of the mandible is completed, the prefabricated cutting guide is fixated to the exposed mandible with screws, and the predictive holes drilled. Finally, the mandible is cut and resected along the predictive cutting lines with a reciprocating saw. If applicable - care should be taken to protect the inferior alveolar nerve and the lingual soft tissue. In certain instances, it is necessary to separate the temporalis tendon from the coronoid process to fully free the specimen. Once the resected mandible has cleared the field, spot hemostasis can be achieved with electrocautery to prep the field for the microvascular anastomosis. Alternatively, an intra oral approach with occlusal based guides can be chosen.

While the ablative team carries out the mandibulectomy, the reconstructive team harvests the fibular flap: The surgeon draws a line from the head of the fibular to the lateral malleolus and incises and dissects down to the fascia of the peroneal muscles. Upon encountering the lateral compartment musculature, dissect it off the fibula from distal to proximal, and then enter the anterior compartment and dissect down to the interosseus membrane, dividing it with electrocautery. This allows enough visibility to make the osteotomies, leaving $7 \mathrm{~cm}$ both distally and proximally to preserve ankle stability and protect the common peroneal nerve respectively. The fibula is then distracted by dissection through the deep posterior muscle compartment, and the peroneal vessels are traced to their origins and transected. Then the surgeon secures the custom cutting guide $7 \mathrm{~cm}$ from the lateral malleolus on the fibular graft and drills the respective predictive holes.

The OMS now uses the selected implant system to carefully complete guided successive osteotomies required for the pre-planned and selected implant sizes according to appropriate manufacture guidelines. Once the DIs have been placed, the fibula osteotomies are completed to create the necessary fibular segments that are then affixed to the custom reconstruction plate to orient the flap into the shape of the reconstructed jaw. At this time, the prosthodontist selects and places appropriate IAs onto the DIs which come in various heights and angle degrees (as described earlier) to correct for skew and create a path of draw for the provisional dental prosthesis. This part is critical, technique sensitive and cumbersome.

After insertion of all the IAs, the prosthodontist secures the provisional abutments to each IA and lutes the provisional prosthesis to the provisional abutments with dual cure composite resin, using a dental dam to maintain a clean surgical field. The prosthodontist utilizes the model of the patient's skull to ensure proper occlusion and delivery of the prosthesis. Once this is verified, the prosthesis is removed and polished for delivery.

The reconstructive surgeon now moves to the head and neck region and completes preparation of the vessels, typically the facial artery and vein for microvascular anastomosis. The fibular pedicle is divided, and the flap is brought to the head and neck region. The graft is then plated to the native mandible with the custom reconstruction plate. Then microvascular anastomosis is performed with 9-0 Nylon for the artery and venous coupler for venous anastomoses. After ensuring adequate flap reperfusion, the head and neck wounds are closed with 3-0 Vicryl for platysma, 4-0 Vicryl for deep dermal, and a 5-0 Prolene stitch for the skin. Intra-orally the excess gingiva is trimmed, and the prosthodontist delivers the provisional prosthesis onto the implants and makes any necessary final adjustments. The soft tissue is sutured closed under the prosthesis to prevent oro-cervical communication.

\section{Post-operative care}

The patient must be closely monitored for several days (typically 5-7) for adequate flap perfusion. Patients will ambulate with physical therapy on post-operative day (POD) 1, and trial a clear liquid diet on POD 3-5. They are discharged on POD 5-7 on a pureed diet, which can be advanced to a mechanical soft diet in 3 
weeks. Eight weeks after their operation they can resume a regular diet. They will follow up with their prosthodontist for frequent outpatient visits and prosthesis adjustments/maintenance and receive their final prosthesis at 4 months.

\section{CASE REPORT}

We performed the procedure as described above in August 2019 [Table 1]. Our index patient was a 51-yearold male who initially presented in 2011 to the treating OMS with an OKC [Figure 4]. At that time, he was treated with enucleation and curettage of the lesion without dental extractions. He was followed closely for 7 months post-operatively and then was lost to follow up until early 2019 when he re-presented upon selfreferral for re-evaluation. The patient had no symptomatic complaints with a past medical history notable only for hypertension that was well controlled. On exam, he demonstrated no facial asymmetry, no obvious swelling, and a slight paresthesia of the mandibular division of the trigeminal nerve on the left side that he reported had been unchanged since his previous surgery in 2011. Intraorally - the patient presented with an intact permanent dentition with no advanced caries or periodontal disease. There was no apparent expansion of the alveolar process and no palpable defect in the buccal or lingual cortex throughout the mandible. Soft tissue changes and scar were noted which were consistent with prior surgery in the area of his lower left quadrant. A 2-D panoramic radiograph was taken which demonstrated a large, mesial-distal extending, multi-loculated, radiolucent lesion [Figure 5]. At that point the sum of the findings of the exam were carefully explained to the patient. Written informed consent was obtained and an incisional biopsy was obtained, with simultaneous placement of a nasal cannula tubing to serve as a drain (given high suspicion for OKC). Histological diagnosis confirmed a recurrence of an OKC. Following extensive discussion with the patient, and all risks and benefits of multiple treatment options were reviewed, the patient elected for definitive surgical resection and reconstruction with a fibula free flap with immediate DI placement and immediate dental restoration. Thus, we planned for segmental mandibulectomy, tooth extractions, right inferior alveolar nerve lateralization, left osteocutaneous free fibular flap reconstruction, reconstruction of the mandibular defect with a custom plate, DI placement, provisional dental prosthesis delivery, and immediate loading of DIs.

Further workup included a computed tomography (CT) maxillofacial with $0.5 \mathrm{~mm}$ slicing per surgical planning company (Medical Modeling Inc., Golden, Colorado) and customized medical device company (Stryker Corporation, Kalamazoo, MI) guidelines [Figure 6]. The patient underwent pre-operative computed tomography angiography of the lower extremities which revealed distal narrowing of the peroneal vessels, more so on the right than the left, so it was decided to harvest the graft from his left side. The patient underwent his operation without major complication [Figure 7]. However of the five planned DIs, only three were placed due to insufficient fibula bone width at the selected sites, an unforeseen planning error. The provisional prosthesis included 10 teeth [Figure 8]. Other than failure to place all planned DIs, he suffered no complications intra-operatively or post-operatively and was discharged seven days later on a puree diet.

He has been seen for continual follow up, through 2021 and is currently undergoing post-surgical modifications in preparation for his definitive prosthesis [Figure 9].

\section{Discussion and review of the literature}

The placement of DIs in a vascularized free tissue flap in preparation for a delayed dental prosthesis has been well described. Implant placement with immediate prosthesis delivery in a single operation has been seldom reported, and is a novel treatment paradigm. The index case above details the practical application of "Jaw in a Day." Since the first description by Levine et al. ${ }^{[1]}$ in 2013 , there have been 20 recorded cases, 
Table 1. Our "Jaw in a Day" experience

\begin{tabular}{|c|c|c|c|c|c|c|c|c|c|}
\hline Age & Gender & Pathology & $\begin{array}{l}\text { Implants } \\
\text { planned }\end{array}$ & $\begin{array}{l}\text { Implants } \\
\text { placed }\end{array}$ & $\begin{array}{l}\text { Teeth restored [Total } \\
\text { (specific teeth)] }\end{array}$ & Complications & Diet & LOS & $\mathbf{F} / \mathbf{U}$ \\
\hline 51 & Male & OKC & 5 & 3 & $10(20-29)$ & $\begin{array}{l}\text { Unplaced } \\
\text { implants }\end{array}$ & Puree & $\begin{array}{l}7 \\
\text { days }\end{array}$ & $\begin{array}{l}24 \\
\text { months }\end{array}$ \\
\hline
\end{tabular}

F/U: Follow up; LOS: length of stay; OKC: odontogenic keratocyst.

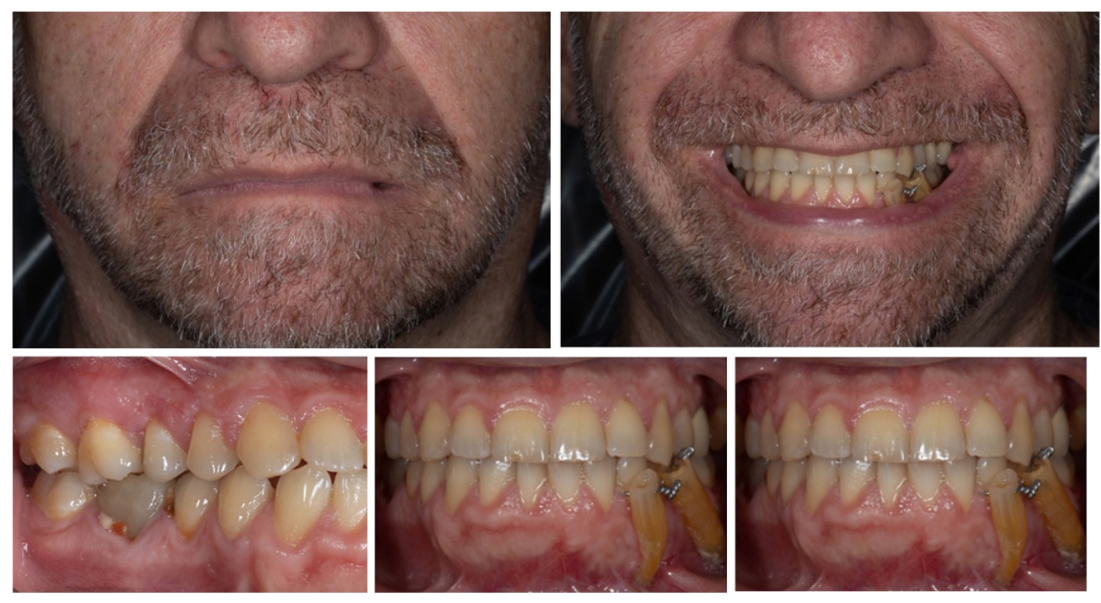

Figure 4. Pre-operative intra and extra-oral photographs. A 51-year-old male with odontogenic keratocyst and good dentition, without facial asymmetry or swelling. Photos are one week status post incisional biopsy and drainage placement.

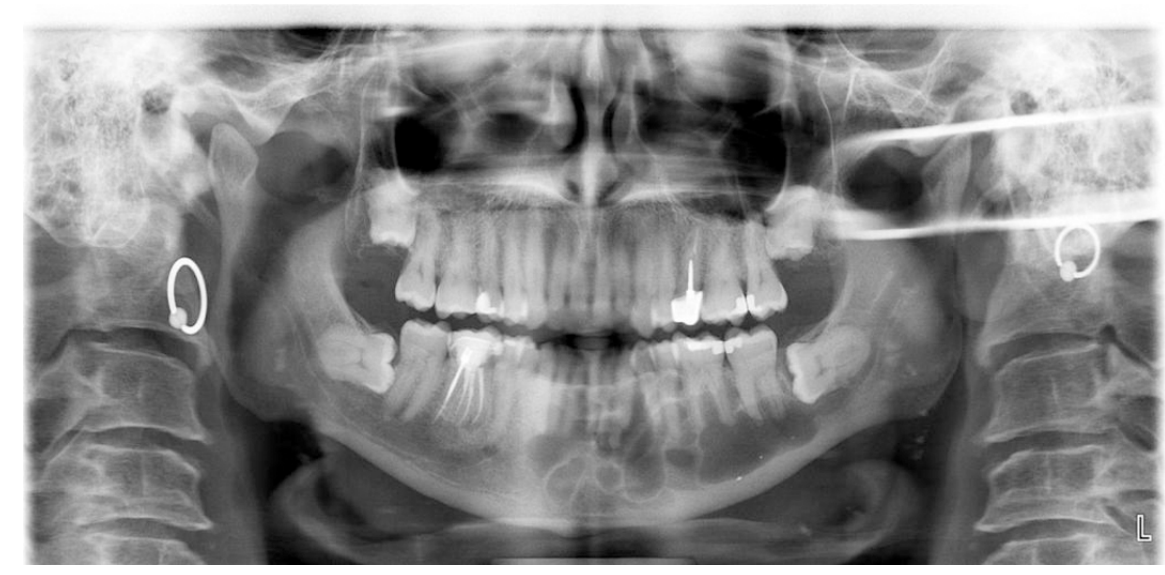

Figure 5. Pre-operative panoramic radiograph at time of re-presentation in 2019.

described in seven series from eight separate institutions 21 total cases inclusive of ours ${ }^{[1-8]}$ [Tables 2 and 3]. Only cases with same-day placement of DIs and an implant-borne provisional prosthesis on a vascularized graft were included in this literature review, conducted via PubMed [Figure 10]; many institutions described immediate DI placement in the original reconstructive surgery followed by implant exposure and prosthesis loaded weeks to months later; these cases were excluded. Isolated cases with immediate prosthesis placement were also culled from larger series reporting on implant placement. 

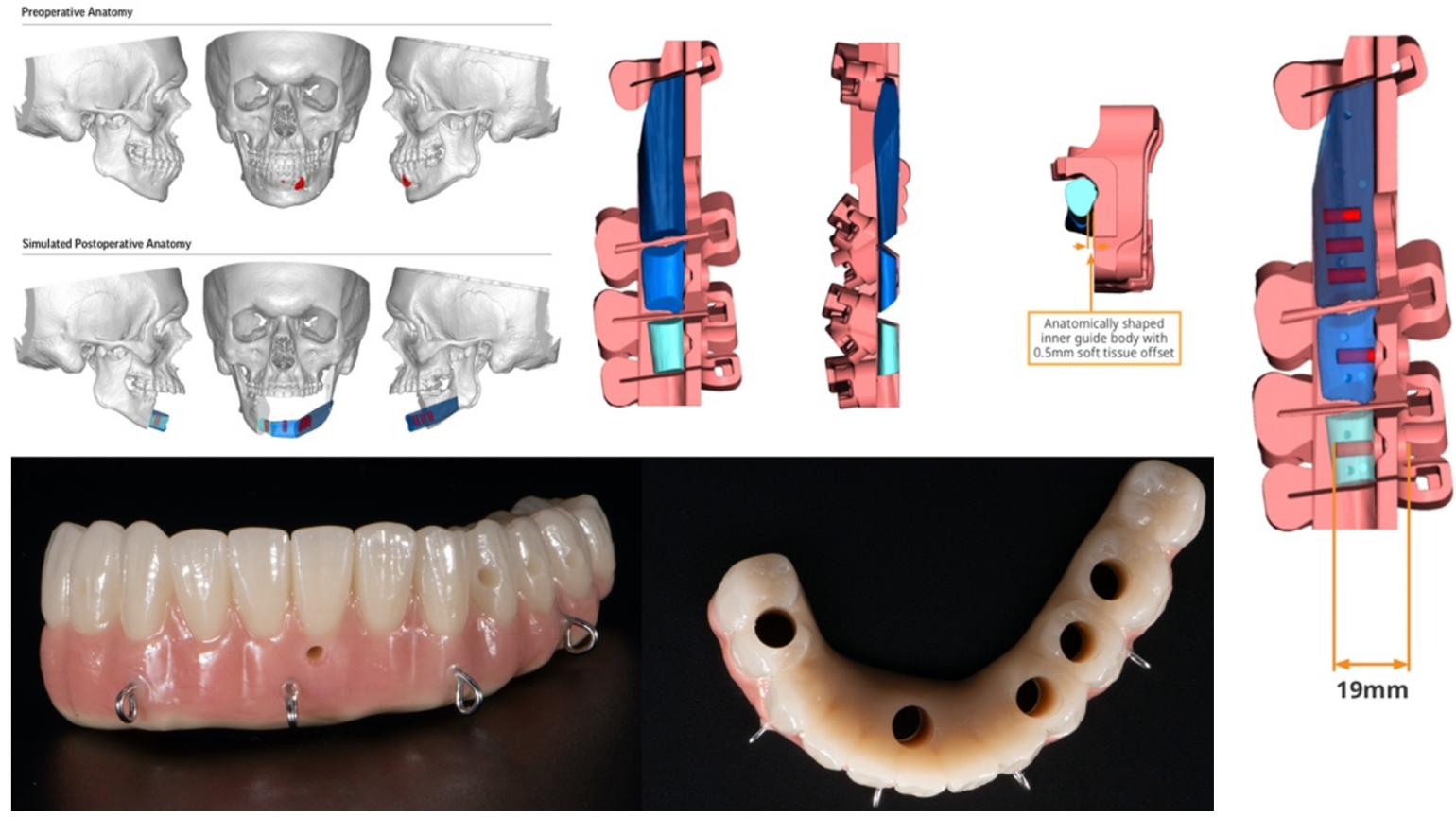

Figure 6. Representative images from the virtual surgical plan and digitally designed and manufactured provisional prosthesis.
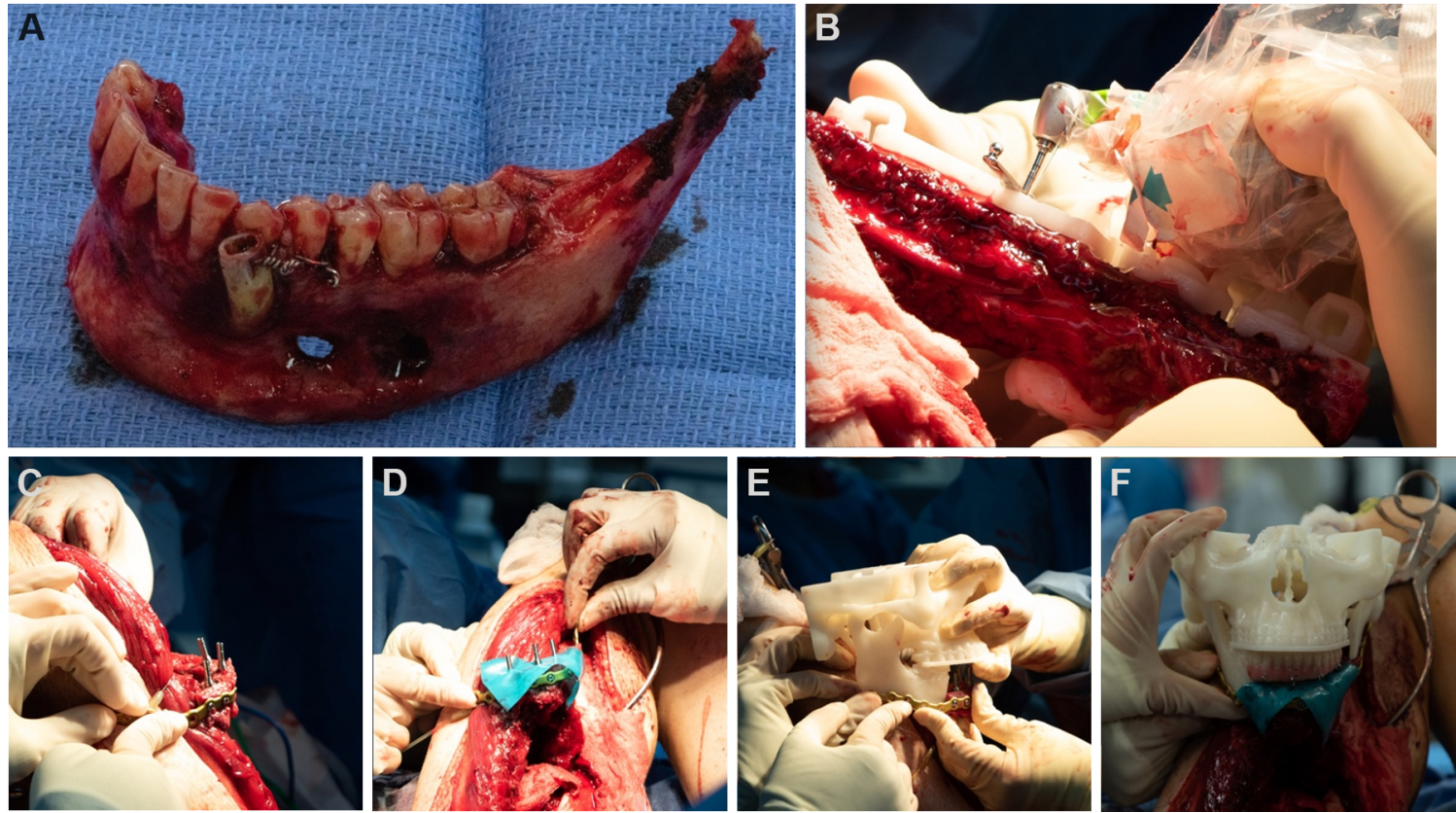

Figure 7. Intraoperative photos. Excised mandible (A), dental implant osteotomy in process (B), intermediary abutment placement (C), application of dental dam to maintain a clean field (D), luting of the provisional prosthesis (E), and occlusion verification with skull model (F).

Of these 21 patients, $11(52.4 \%)$ were male and 10 (47.6\%) were female. Patients ranged in age from 16-76 years old (averaged 35.1 years). Three cases (14.3\%) performed were for oncologic resection with squamous cell carcinoma identified as the most common pathology. Eighteen cases (85.7\%) were performed in cases of patients with benign pathology including ameloblastoma, AV malformation, eosinophilic granuloma, 


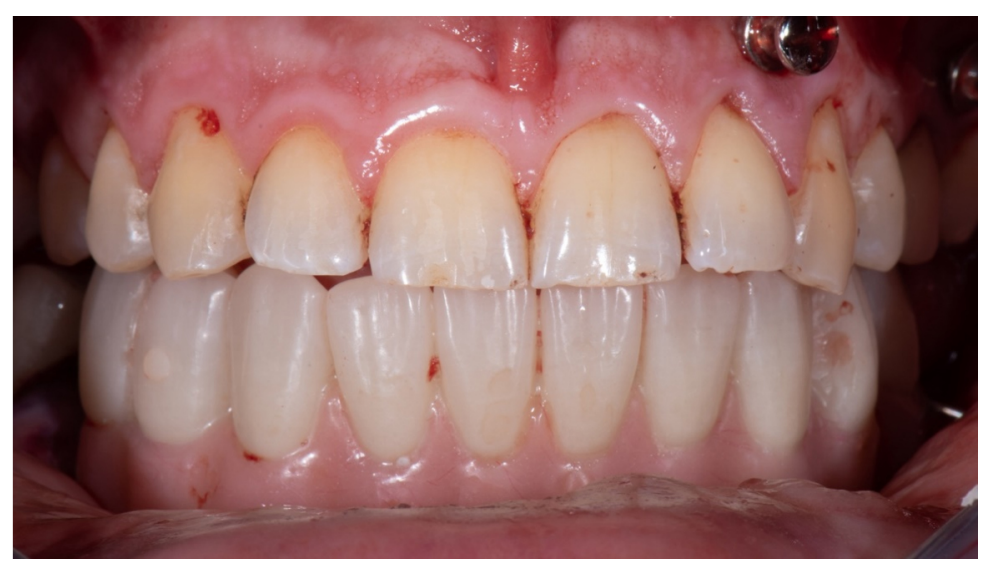

Figure 8. Post-operative anatomy. Proper occlusion and able to tolerate a normal diet.
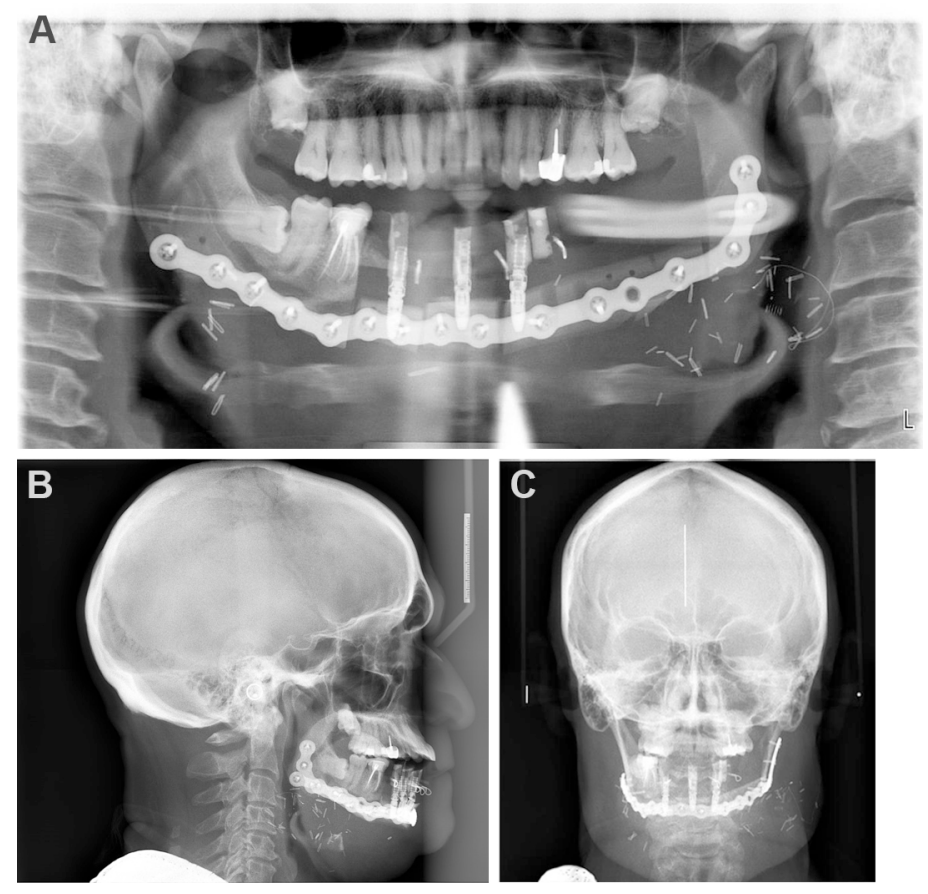

Figure 9. Post-operative demonstrating panoramic radiograph (A), lateral cephalogram (B), and PA cephalogram (C).

Table 2. Patient demographics of recorded "Jaw in a Day" procedures ${ }^{[1-8]}$

\begin{tabular}{|c|c|c|c|c|c|c|c|}
\hline Ref. & Institution & Year & $n$ & Gender & Age (mean) & Age (range) & Pathology \\
\hline Levine et al. ${ }^{[1]}$ & NYU Langone & 2013 & 4 & $2 \mathrm{M}, 2 \mathrm{~F}$ & 25.75 & $20-34$ & $4 B$ \\
\hline Meloni et al. ${ }^{[4]}$ & University Hospital of Sasari Italy & 2015 & 6 & $3 \mathrm{M}, 3 \mathrm{~F}$ & 55.5 & $36-70$ & $3 \mathrm{C}, 3 \mathrm{~B}$ \\
\hline Qaisi et al. ${ }^{[2]}$ & University of Mississippi & 2016 & 3 & $2 \mathrm{M}, 1 \mathrm{~F}$ & 44.3 & $23-76$ & $3 B$ \\
\hline Runyan et al. ${ }^{[7]}$ & NYU Langone, MSK, Lenox Hill & 2016 & 1 & $1 \mathrm{~F}$ & 26 & 26 & $1 \mathrm{~B}$ \\
\hline Lee et al. ${ }^{[3]}$ & NYU Langone & 2018 & 2 & $2 \mathrm{M}$ & 22.5 & $19-26$ & $2 B$ \\
\hline Grewal et al.* & Columbia NYP & 2019 & 1 & $1 \mathrm{M}$ & 51 & 51 & $1 \mathrm{~B}$ \\
\hline Patel et al. ${ }^{[6]}$ & Providence Cancer Institute & 2019 & 2 & $2 \mathrm{~F}$ & 19.5 & $19-20$ & $2 B$ \\
\hline Sukato et al. ${ }^{[8]}$ & SUNY Downstate & 2020 & 2 & $1 \mathrm{M}, 1 \mathrm{~F}$ & ND & ND & $2 B$ \\
\hline Totals & & & 21 & $11 \mathrm{M}, 10 \mathrm{~F}$ & 35.1 & $16-76$ & $3 \mathrm{C}, 18 \mathrm{~B}$ \\
\hline
\end{tabular}


"Our case report. B: Benign; C: cancerous; F: female; M: male; ND: not disclosed.

Table 3. Patient outcomes of recorded "Jaw in a Day" procedures ${ }^{[1-8]}$

\begin{tabular}{|c|c|c|c|c|c|c|c|c|c|c|}
\hline Ref. & $n$ & $\begin{array}{l}\text { Median \# } \\
\text { implants }\end{array}$ & $\begin{array}{l}\text { Total \# } \\
\text { implants }\end{array}$ & $\begin{array}{l}\text { Implants } \\
\text { lost }(n, \\
\%)\end{array}$ & $\begin{array}{l}\text { Median \# } \\
\text { teeth } \\
\text { restored }\end{array}$ & $\begin{array}{l}\text { Total \# } \\
\text { teeth } \\
\text { restored }\end{array}$ & $\begin{array}{l}\text { Dentition } \\
\text { spatiality } \\
\text { (teeth \#s) }\end{array}$ & $\begin{array}{l}\text { Post-op } \\
\text { dietary } \\
\text { protocol }\end{array}$ & $\begin{array}{l}\text { LOS } \\
\text { range } \\
\text { (days) }\end{array}$ & $\begin{array}{l}\text { F/U range } \\
\text { (months) }\end{array}$ \\
\hline Levine et al. ${ }^{[1]}$ & 4 & 5 & 21 & 0 & 9 & 35 & $\begin{array}{l}\text { L Md (18-30) } \\
\text { R Mx (2-10) } \\
\text { Md (19-29) } \\
\text { Md } 4 \text { teeth } \\
\text { NOS }\end{array}$ & ND & $8-29$ & $10-22$ \\
\hline Meloni et al. ${ }^{[4]}$ & 6 & 5 & 29 & 3 & ND & ND & $\begin{array}{l}\text { Md A x } 3 ; \mathrm{R} \\
M n ; M \times A ; L \\
M n\end{array}$ & ND & ND & 48 \\
\hline Qaisi et al. ${ }^{[2]}$ & 3 & 4 & 13 & 0 & 6 & 19 & $\begin{array}{l}\text { R Md (25-29) } \\
\text { Md (21-28) } \\
\text { R Md (26-31) }\end{array}$ & $\begin{array}{l}\text { Puree diet for } 3 \\
\text { months }\end{array}$ & ND & $4-17$ \\
\hline Runyan et al. ${ }^{[7]}$ & 1 & 3 & 3 & 0 & 8 & 8 & $\mathrm{R} M \times(2-9)$ & $\begin{array}{l}\text { Tube feed for } 5 \\
\text { days; soft diet }\end{array}$ & 5 & 5 \\
\hline Lee et al. ${ }^{[3]}$ & 2 & 3 & 6 & 0 & ND & ND & ND & $\begin{array}{l}\text { Tube feeds } \times 2 \\
\text { weeks; liquids } \times \\
3 \text { weeks; soft } \\
\text { diet } \times 1 \text { week }\end{array}$ & ND & $18.9-38.3$ \\
\hline Grewal et al. ${ }^{*}$ & 1 & 3 & 3 & 0 & 10 & 10 & Md (20-29) & Puree** & 7 & 24 \\
\hline Patel et al. ${ }^{[6]}$ & 2 & 3.5 & 7 & 0 & 6 & 12 & $\begin{array}{l}\text { L Md (19-27) } \\
\text { R Mx (1-4) }\end{array}$ & ND & ND & ND \\
\hline Sukato et al. ${ }^{[8]}$ & 2 & 6 & 12 & 0 & 12 & 24 & $\begin{array}{l}\text { Md (19-30) } \\
\times 2\end{array}$ & $\begin{array}{l}\text { Soft diet for } 24 \\
\text { h }\end{array}$ & ND & ND \\
\hline Totals & 21 & & 94 & $3(3.2 \%)$ & 8.5 & 108 & & & $5-29$ & $5-48$ \\
\hline
\end{tabular}

"Our case report. "See case report for more details. \#: Number; A: arch; F/U: follow up; L: left-side; LOS: length of stay; Md: mandible; Mx: maxilla; ND: not documented; OF: osseointegration failure; R: right-side; NOS: not otherwise specified.

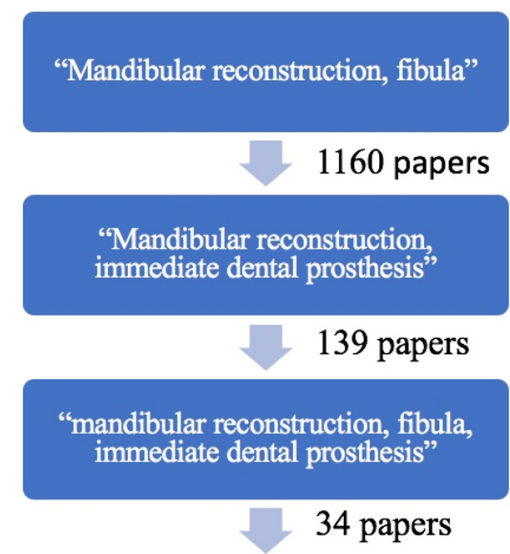

\section{Exclude studies in which provisional prosthesis was not} loaded immediately, at time of resection

\section{7 papers}

Figure 10. PubMed search terms used in the literature review to find cases of "Jaw in a Day" procedures.

gunshot wound, mucormycosis, odontogenic myxoma, osteoblastoma, and osteomyelitis. Only Qaisi et al. ${ }^{[2]}$ s 2016 case series commented on comorbidities; one of their three patients had well-controlled 
diabetes mellitus II and hypertension.

There were no reported cases of flap failures or other surgical complications requiring re-operation. Inclusive of all cases reported, a total of 41 implants were placed overall with a median of four implants per patient. Of these, 3 (3.2\%) were lost secondary to failed osseointegration. Centers did not report on planned versus placed implants, so the frequency of this planning error cannot be ascertained. Of the series which reported the number of teeth restored, a median of 8.5 teeth were restored per patient. Most of the case series did not directly report on the spatial placement of restored dentition; however, laterality and which exact teeth were restored were able to be determined from photographs of the provisional prostheses included in the papers. This operation has been recorded on both sides and the arch of both the maxilla and mandible.

In terms of dietary consistency and transition, Qaisi et al. ${ }^{[2]}$ enforced a strict puree diet for 3 months; Runyan et al. ${ }^{\left[{ }^{[}\right]}$'s patient received tube feeds for five days post-operatively, then was transitioned to a soft diet; Lee et al. ${ }^{[3]}$ 's dietary protocol included two weeks of tube feeds, three weeks of liquids, and one week of a soft diet before resumption of normal diet; and Sukato et al.$^{[8]}$ maintained a $24 \mathrm{~h}$ soft diet protocol. The average postoperative stay was 14 days (5-29 days). Follow-up ranged from 5-48 months. On last follow-up, all patients demonstrated adequate occlusion, satisfactory aesthetics, and were able to tolerate a normal diet.

Costs associated with DIs, and prosthesis design and production, as well as funding sources were not detailed in any of the series. Those that did mention cost, did so only to mention it represented a significant impediment to wider spread implementation. Certainly, it is a limitation of the current healthcare system to consider replacement of lost teeth as a cosmetic procedure following extensive surgery for benign and malignant disease. To a patient, restoring the oral cavity to their original form, and function, in addition to allowing them to smile may be the most important factor for success following this surgical reconstruction.

The review of the literature demonstrates the apparent success and growing implementation of such procedures that can restore a patient's oral and aesthetic jaw function in a single day. It is undoubtedly an important and impactful treatment option to offer to select patients who meet criteria. Further, although research is still limited, there do not appear to be immediate detrimental consequences to either flap success or an increase in intra-operative complications. The summation of JIAD literature report only three implant failures, for a $96.8 \%$ success rate. This compares favorably to Okay et al. ${ }^{[5]}$, which observed an $89.3 \%$ dental implant success rate in their 13 patients who underwent delayed DI placement with immediate prosthesis placement (50 of 56 implants) and an $81.7 \%$ success rate of 15 patients who underwent delayed DI placement and delayed prosthesis placement (49 of 60 implants).

However, such conclusions are dampened by the nature of the retrospective reviews. This review is limited by inconsistencies in the reporting of demographic data, comorbidities, complications, and outcomes. Notably, none of the current papers make an effort to describe the expense associated with such a complicated procedure; in fact, only two mentioned the burden of cost at all.

Further research is required on patient satisfaction, and the short-term or long-term success of not only the free flap, but the success of immediately placed DIs when compared to a delayed protocol, and immediate versus delayed delivery of a dental prosthesis. Nonetheless, "Jaw in a Day" procedures are an interdisciplinary feat of technological planning and surgical teamwork that, when done right, can greatly benefit patients. It is this team's hope that by describing the case study, and literature, and standardizing a protocol - it will help to make this revolutionary advancement more easily achievable for other institutions 
and more accessible for patients in the future.

\section{DECLARATIONS}

\section{Authors' contributions}

Made substantial contributions to conception and design of the study and performed data analysis and interpretation: Grewal M, Forman MS, Song SJ, Troob S

Performed data acquisition, as well as provided administrative, technical, and material support: Grewal M, Forman MS, Song SJ, Troob S, Eisig S, Friedman J

\section{Availability of data and materials}

Not applicable.

\section{Financial support and sponsorship}

None.

\section{Conflicts of interest}

All authors declared that there are no conflicts of interest.

\section{Ethical approval and consent to participate}

Not applicable.

\section{Consent for publication}

All authors have given their consent for publication.

\section{Copyright}

(c) The Author(s) 2021.

\section{REFERENCES}

1. Levine JP, Bae JS, Soares M, et al. Jaw in a day: total maxillofacial reconstruction using digital technology. Plast Reconstr Surg 2013;131:1386-91. DOI PubMed

2. Qaisi M, Kolodney H, Swedenburg G, Chandran R, Caloss R. Fibula Jaw in a Day: state of the art in maxillofacial reconstruction. J Oral Maxillofac Surg 2016;74:1284.e1-1284.e15. DOI PubMed

3. Lee ZH, Avraham T, Monaco C, Patel AA, Hirsch DL, Levine JP. Optimizing functional outcomes in mandibular condyle reconstruction with the free fibula flap using computer-aided design and manufacturing technology. J Oral Maxillofac Surg 2018;76:1098-106. DOI PubMed

4. Meloni SM, Tallarico M, De Riu G, et al. Guided implant surgery after free-flap reconstruction: four-year results from a prospective clinical trial. J Craniomaxillofac Surg 2015;43:1348-55. DOI PubMed

5. Okay DJ, Buchbinder D, Urken M, Jacobson A, Lazarus C, Persky M. Computer-assisted implant rehabilitation of maxillomandibular defects reconstructed with vascularized bone free flaps. JAMA Otolaryngol Head Neck Surg 2013;139:371-81. DOI PubMed

6. Patel A, Harrison P, Cheng A, Bray B, Bell RB. Fibular reconstruction of the maxilla and mandible with immediate implant-supported prosthetic rehabilitation: Jaw in a Day. Oral Maxillofac Surg Clin North Am 2019;31:369-86. DOI PubMed

7. Runyan CM, Sharma V, Staffenberg DA, et al. Jaw in a Day: state of the art in maxillary reconstruction. J Craniofac Surg 2016;27:2101-4. DOI PubMed PMC

8. Sukato DC, Hammer D, Wang W, Shokri T, Williams F, Ducic Y. Experience with "Jaw in a Day" technique. J Craniofac Surg 2020;31:1212-7. DOI PubMed 